\title{
La locura de Horacio Quiroga
}

Al describir el suicidio de Horacio Quiroga, Elías Castelnuovo, un amigo del famoso cuentista, escribe lo siguiente: "El jueves por la tarde salió del hospital donde se encontraba internado para preparar su viaje a Misiones, y el viernes por la mañana (I9 de febrero, 1937) emprendía, en cambio, el viaje sin retorno a la región de las tinieblas. Adquirió una dosis de cianuro y hacia la madrugada, en la soledad de su pieza, sin luz y sin testigos, se envenenó. A fuerza de experimentar en vida, tal vez el horror a la muerte, al llegar el instante de la ruptura, se ve que el hombre sintió una alegría extraña, porque murió con una sonrisa en la boca... Su rostro, blanco, pecoso, denota una tranquilidad absoluta. Yo lo observo así tendido, duro, flaco, con el mismo respeto que me inspiró en vida. Con la misma seriedad. Más: guardando la misma distancia". (I) Estas condensadas líneas expresan perfectamente ese lado del carácter y la naturaleza del fin de Horacio Quiroga que vamos a estudiar en nuestro análisis de su locura vista por las páginas de sus obras.

En el mismo artículo Castelnuovo, para explicar el suicidio -acto final de desengaño absoluto - menciona cuatro muertes violentas que Quiroga había atestiguado en el curso de su vida.

(1).-La tragedia de Horacio Quiroga, por Elías Castelnuovo, Claridad, Buenos Aires, marzo de 1937. Reproducido en Repertorio Americano, San José de Costa Rica, I.7 de abril de I937. 
Cuando tenía seis meses, mientras la madre lo amamantaba, un dia, le trajeron al padre muerto de tres tiros de escopeta. A los trece años, el padrastro, que fué el único padre que conoció el muchacho, otro día, en su presencia, se suicidó. Cuando tenía veinte años Quiroga mató accidentalmente a su mejor amigo, Federico Ferrando, con una pistola. $\mathrm{Y}$ finalmente, se casó con una mujer que también se suicidó en presencia suya.

Teniendo en cuenta esta red de circunstancias que al principio de su vida hizo del pobre Quiroga un caso patológico, el lector al examinar sus obras no puede menos de buscar en ellas algún elemento de anormalidad patológica o de incipiente locura que reflejara estas influencias. En efecto, desde la aparición de su primer libro, Los arrecifes de coral en Igor, hasta su última colección de cuentos Más allá, 1935, el autor demuestra cada vez más esta propensión hacia lo anormal, la Jocura y la muerte. Su única fe valedera fué en un eterno amor.

En los primeros libros de nuestro autor la influencia de Poe es obvia, y como en el caso de Poe, Quiroga como escritor mantiene en su juventud cierta objetividad que no le deja identificarse completamente con sus personajes. Sin embargo, estos son un reflejo del frustrado y adusto carácter de su creador. Los arrecifes de coral, por ejemplo, contiene un cuento titulado EL GUARDABosoue comediante en que el loco protagonista se repetía la frase "yo soy romano y negligente", y otras necias frases por el estilo, hasta que al fin sale de su cabaña en pleno invierno y es devorado por una bandada de lobos. Otro cuento en el mismo libro, Sin Razón, pero CANSADO, describe el hastío del amor físico y la muerte de la amada a manos del amante. El marido de la mujer, muy amigo del asesino, al enterarse de la muerte de su esposa sólo pregunta: "Hizo mucho resistencia?" $\mathrm{Y}$ el asesino contesta con un distraído: "Mucha", terminando así el relato. En este libro ambiente, personajes, argumentos, todos están "dibujados como a través de un vitral, y parecen contados por una voz lejana y displicente que llega a nuestros oídos entrecortada y temblorosa..." (I)

(1) --Prólogo de Cuentos, Horacio Quiroga, Montevideo, 1937, por Alberto Lasplaces. 
La segunda obra de Quiroga, El crimen del otro, 1904, revela un desarrollo psicológico más maduro y artístico de parte del autor, quien no obstante sigue manteniendo la distancia del escritor que no ha acabado de descubrirse a sí mismo. En Flor DE IMPERIO, cuando se mtiere Divina, adorada her.. manita de Rubén, éste se siente obsesionado, aplastado por la pérdida. Y cuando finalmente se enamora de una muchacha, "todo su amor de hombre naufragaba en el deseo de ser llorado como una no manchada novia". Para lograrlo agota en sus labios tu ancho frasco de morfina, y muere sonriendo. Los personajes de este cuento son pálidos fantasmas comparados con los intensos amantes que se suicidan en uno de los últimos relatos de Quiroga, MÁs ALLÁ, pero aun así nos descubren las no muy sanas preocupaciones del joven cuentista. La HisTORIA DE EsTILICón, inspirada probablemente en THE MURDERS IN THE RUE MORGUe de Poe, presenta por protagonista a un gorila apasionado de tuna mujer, quien admite sus avances y poco a poco muere de su efecto. El novio ultrajado, en un arrebato de furia azota a la bestia $y$ es violentamente hecho pedazos. Este relato es horripilante sin ser ni muy claro ni coherente. En la misma colección, Er CRIMEN DEL OTRo, sugerido por THE CASK OF AMONTILlado de Poe, repite el cuento del gran cuentista yanqui con la única excepción de que el enterrado (Fortunato), ebrio y obsesionado por la historia de Poe, se identifica con el Fortunato del relato norteamericano hasta tal punto, que entra en el pozo de muy buena voluntad.

La tercera obra de esta primera época en la vicla de nuestro escritor, Los perseguidos, I905, ( I) es una novela corta acerca de dos hombres que se creen perseguidos el uno por el otro. Si no fuese por el terror espantoso que estos dos nersonajes sienten, la historia resultaría ligera y hasta graciosa. Fn esta narración Quiroga no sólo escribe sobre dos perseguidos, sino que presenta un estudio metódico y científico de esta fase de la

(1).-Doy la fecha citada por Lasplaces, op. cit. La primera edición que yo he visto es de 1908 . 
locura. El lector siente la realidad relatada sin dudar por un momento que se trata de algo que realmente ha ocurrido. La interpretación de los sentimientos de los perseguidos es tan fuerte que a veces casi llegamos a creer que Quiroga está apuntando sus propias reacciones:

"Díaz Vélez continuaba caminando y pronto estuve a dos pasos detrás de él. Uno más y lo podía tocar. Pero al verlo así sin darse ni remotamente cuenta de mi inmediación, a pesar de su delirio de persecución y sicologías, regulé mi paso exactamente con el suyo. ¡Perseguido! ¡Muy bien!... Me fijaba detalladamente en su cabeza, sus codos, sus puños, un poco de fuera, las arrugas transversales del pantalón en las corvas, los tacos, ocultos y visibles sucesivamente. Tenía la sensación de que antes, millones de años antes, yo había hecho ya eso: encontrar a Díaz Vélez en la calle, seguirlo, alcanzarlo - y una vez seguir detrás de él- detrás. Irradiaba de mí la satisfacción de diez vidas enteras que no hubieran podido nunca realizar su deseo..."

En esto el primer perseguido empieza a temer que el segundo vaya a volverse y descubrirle:

"Tuve un momento de angustia tal que me olvidé de ser él todo lo que veía: los brazos de Díaz Vélez, las piernas de Díaz Vélez, los pelos de Díaz Vélez, la cinta del sombrero de Díaz Vélez, la trama de la cinta del sombrero de Díaz Vélez, la urdimbre de la urdimbre de Díaz Vélez..."

$\mathrm{Y}$ así sucesivamente hasta que el lector mismo tiene ganas de doblar la cabeza para yer si es perseguido. En los susodichos relatos de El crimen del otro y en Los perseguidos, el autor escribe en la primera persona, y casi siempre figura como tuno de los personajes principales en el argumento. Ninguna de las narraciones de esta primera época de Quiroga prueba que el autor está en camino de volverse loco, pero todas ellas revelan una extremada admiración que a veces llega a ser una obsesión por la locura, y por las pesadillas poeanas. Desafortu1- 
nadamente, el impresionable Quiroga nunca pudo deshacerse de estos primeros lúgubres choques e inclinaciones que dejaron huellas indelebles en su cerebro y en su personalidad.

En r9ro Quiroga y su esposa se avecindan en Misiones, y el autor sigue escribiendo para revistas de Buenos Aires, sin publicar ninguna colección de ctientos hasta I9I7, cuando aparecen los Cuentos de amor, de locura y de muerte. (I) Su vida allá en el pueblo de San Ignacio fué bastante activa para dar equilibrio a su inquieto espíritu, y el resultado es una serie de seis libros de hermosos y variados cuentos que abarcan lo mejor de su producción literaria. (2) La primera de estas colecciones, la que acabamos de citar, contiene dos cuentos negros: LA GALLINA DEGOLLADA, en que los cuatro hijos idiotas de una familia desangran a su hermanita normal como se degüiella a una gallina, y EL ALMoHAdón DE PLUMA, historia de una mujer enferma cuya sangre es chupada por un enorme y monstruoso parásito de las aves ocultado en su almohadón. LA MIEL SILVESTRE no describe ninguna monstruosidad anormal, pero presenta el cuadro horripilante de un hombre que es paralizado por algún veneno en la miel silvestre que engulle para quedar después consciente e inmóvil ante un enjambre de hormigas que avanzan sobre él para devorarle. Otros cuentos de esta colección en que la muerte ocupa un sitio importante son: A EA DERIVA, interpretación objetiva de la muerte por mordedura de víbora en que la naturaleza contempla con fría indiferencia la tragedia del hombre, LA INSOLAcrón, fantástica historia de la muerte vista y prevista por los perros del protagonista quien sin saberlo está próximo a morir, EL solitario, venganza de un manso y abusado joyero que mata a su esposa infiel con un alfiler de brillantes después que ella le llama "aburrido cornudo", y Los BUQUES suICIDAN-

(I) - Vencido por la muerte súbita de su mujer y otras pérdidas, Quiroga regresa a Buenos Aires en 1917 para dar educación escolar a sus criaturas. Enrique Espinoza, Trayectoria de Horacio Quiroga, en la revista Atenea, febrero de 1937. .

(2).-Cuentos de amor, de locura y de muerte; Cuentos de la selva; El salvaje; Anaconda; El desierto; Los desterrados. 
TES, en que el autor presenta un grupo de hombres llevados al suicidio por el propio terror que tienen a la posibilidad de suicidarse. Este último relato conserva una vena casi casual que todavía demuestra la objetividad dil escritor; los otros relatos mencionados vuelven a sugerir a Poe, pero la técnica es fría y lógica como una hoja de acero, y nos convence de que Quiroga es maestro de su pluma sin ser necesariamente uña y carne con ella. Debiéramos añadir que en esta misma colección hay dos narraciones largas y ligeras - una de ellas humoristica- que son de las mejores que nuestro autor ha escrito en este género: EL PRIMER CIGARRo y LA MENINGITIS Y SU sombra. Son la antítesis de los relatos que acabamos de comentar, y con su saludable contraste dan una prueba más del equilibrio mental del escritor.

El próximo libro de Quiroga, Cuentos de la selva, (para niños), 1918, revela otro lado de su carácter: el amor por los niños y un profundo conocimiento de su psicología. (I) No hay en toda la colección ni el más leve indicio de anormalidad. Esto nos lleva a creer que el autor ha pasado de la edad de su juvenil sentido de fracaso y horror. El salvaje, publicado en 1920, da más pábulo a esta idea a pesar de que contiene dos o tres narraciones bastante naturalistas. UNA BOFETADA describe la horrible venganza de un peón quien para desquitarse de un hombre que le había dado una bofetada. le azota sin misericordia forzándole a caminar por la selva hasta que se desploma y expira. "Y el rebenque, con terrible y monótona violencia cayó sin tregua sobre la cabeza y la nuca de Korner, arrancándole mechones sanguinolentos de pelo". LOS IN MIGRANTES, LOS CEMENTERIOS BELGas y LA REINA ITALIANA, también contienen pasajes de una vividez casi empalagosa, mientras que Estefanía y La llama analizan con meticuloso cuidado el desarrollo de casos psicológicos anormales. Estefanía, una muchacha joven y bonita, se suicida después de fracasar dos veces en sus amores, y en LA LLAMA una niña de diez se envejece treinta años al escuchar en un arrebato de extre-

(I) --EI PRIMIR CIGARRo también pertenece a este género. 
mada pasión la partitura de Tristán e Isolda que dura sólo hora y cuarto. En todos estos relatos, con la posible excepción de Los emigrantes, el naturalismo y el sentido de la anormalidad están controlados y subordinados al desarrollo artístico. Otros cuentos en el mismo libro: Tres cartas $\mathrm{y}$ UN PIE, Cuento para Novios y UN IDILIO son sanos bosquejos humorísticos.

En Anaconda, I921, si excluímos la breve narración titulada EL YaCryatere, basada en una superstición más bien que en una anormalidad, Quiroga abandona completamente el tema de la psicología patológica. E1 yaciyateré es un pajarraco desgarbado que canta de noche y que vienè a llamar a un chiquilín enfermo de meningitis para dejarlo loco. En EL sIMÚN y GLORIa TROPICAL el escritor revela su afición a los extremos del tiempo -afición que se nota en casi todas sus narraciones en que el tiempo se menciona. El frío es siempre frío que traspasa; el calor, de 48 grados: a la sombra, donde no hay más que arena y escorpiones; las lluvias, verdaderos temporales de agua que se asientan sobre el bosque. En El srmún encontramos esta descripción del sirocco: "Cuando sopla el sirocco, si no quiere usted estar todo el día escupiendo sangre, debe acostarse entre sábanas mojadas, renovándolas sin cesar porque se secan antes de que usted se acuerde. Así dos, tres días. A veces, siete..." Gloria tropical describe el crecimiento rápido, fenomenal de las plantas en Fernando Poo cerca de la boca del Níger en Africa. Estos dos cuentos, y varios otros por el estilo, indican si no prueban que Quiroga no pudo ni quiso mantener la justa proporción de las cosas cuando le entraba uno de esos caprichos por la desproporción. Y el suicidio no es más que el resultado de una actitud desproporcionada ante la vida. Debiéramos agregar que Polea loca en el mismo libro es de un humor tan claro y vital que el lector de solo este cuento diría que el autor era el hombre más sano del mundo.

El desierto, 1924, contiene más de la filosofía del Quiroga normal que ningún otro libro stuyo, y en él encontramos sus páginas más profundas. Los tres resos, Ex potro salvaje, EL 
LEón, JUAN DARIÉN, y sobre todo, LA PATRIA, son alegorías que reflejan $\mathrm{y}$ abarcan los pensamientos $\mathrm{y}$ sentimientos de un espíritu moderado, justo, sincero y compasivo. Tal vez esta misma compasividad, extremada sensibilidad y tolerancia que tuvieron que sufrir tanto en un mundo de crueldades e intolerancias, lograron por fin destrozar la fuerza y el delicado equilibrio que luchaban por sobrevivir en el cuerpo no muy fuerte de escritor. Todas estas alegorías son claras y vibrantes, mientras que en el mismo libro los dos relatos que giran sobre lo irracional son débiles e incoherentes. Tanto EL síNCOPE BLANCO como EL ESPECTRO tratan de ese terreno limítrofe entre la vida y la muerte donde las almas se revelan como a través de un vitral y llegan a comprender los dos mundos por el puente del amor. EL DEsIERTo, que es el cuento más tierno del escritor, tiene algunos párrafos sobre la muerte, pero en ellos Quiroga demuestra un gran avance sobre sus esfuerzos anteriores tratando del mismo tema. La muerte del padre en EL DESIERTo cumple el propósito que todo elemento de cuento debiera cumplir, es decir, ocupa un sitio necesario en el cuadro completo sin destruír ni en lò más mínimo la perspectiva del producto artístico en su conjunto. (I)

Al contrario, dos años más tarde, en Los desterrados, I926, la muerte ocupa un lugar si no ubicuo al menos principal en todos los ocho relatos del libro, excepto en EL TECHO DE INCrENSo, breve narración humorística. La muerte no sólo hace un papel principalísimo en estos cuentos, sino que se presenta con todos sus tonos y matices: tierna, grotesca, natural, filosófica, horrible, fea y hasta loca y alcohólica. Sólo el vigor del ambiente y de los personajes misioneros, nunca tan fuertes como en estas pulsantes páginas, salva al libro de la obsesión extravagante de un espíritu enfermo y mórbido. Sin embargo, estos

(1).-En I925 el autor publica La gallina degollada y otros cuentos que contienen sólo un relato nuevo, EL perro rabroso, historia de un hombre que se vuelve loco y dispara a todo el mundo con la alucinación de que están atacándole millares de víboras. Los vecinos lo rastrean en el monte y le hallan trepado en un árbol aullando de un modo horrible. Le matan de un tiro. 
cuadros tienen su otro lado. En la Cámara oscura, por ejemplo, después de darnos asco con su repugnante descripción de un hombre muerto, el autor al parecer siente remordimiento, y algunas páginas más allá escribe del narrador: "Al salir afuera, la noche libre me dió la impresión de un amanecer cargado de motivos de vida y de esperanzas que había olvidado: flores, valle, río, vaga niebla, bosque". A pesar de éste y unos cuantos otros pasajes semejantes, y a pesar de varias páginas recortadas con agudos toques de pluma afilada, el efecto total de Los desterrados es de depresión, hastío, fealdad y muerte casi nauseabundos.

La segunda novela de Quiroga, Pasado amor, 1929, vale poco como novela, pero en ella se descubren muchos detalles que parecen sacados vibrantes de la vida del autor. En breve, el argumento es como sigue: Morán, viudo, ama a Magda, una muchacha joven, quien a instancias de su madre le rechaza por su ateísmo. Morán, a su vez, es amado perdidamente por Alicia Hontou a quien había adulado distraídamente una noche de fiesta, y quien al encontrarse abandonada por él, se envenena. Las mejores páginas del libro son las últimas en que el universo se desploma sobre Morán que no prevé ni puede prever el acontecimiento fatal que va a aniquilarlo. "Nada en el cielo, ni en las cosas miradas, ni en la tierra hollada, advierte al hombre que el universo se desplomará sobre él. Sigue su camino, dichoso de existir: grato a las cosas que to contemplan, al perfume de los azahares del monte que lo exaltan, seguro de poder sonreír a solas, si quiere, pues nadie como él ha redimido y asegurado su vida por medio de un grande y eterno amor". El último capítulo de la novela es la parte más aplicable a la vida del autor. Viene inmediatamente después que Morán se embarca para marcharse de Misiones afirmando que no sabía cuando volvería. Cito el capítulo entero: "Lo sabía, sin embargo. Desde la borda del vapor, que sin pitar y bajo la lluvia cerrada parecía huir también para siempre de Misiones, Morán dirigió los ojos por sobre el monte brumoso hacia el pueblo de la yerba mate, con su fiebre de ganancia que llenaba todo el país, y que para él no encerraba sino dos amores ba- 
jo los cuales, como la sombra del capote que lo velaba, yacía muerto él. Y no sólo él...

"Deseó, ofreció, confió su vida trunca a una felicidad redentora: La religión, más fuerte que un grande y puro amor, se la había negado.

"Cerró los ojos, rehuyó, negó esa misma vida suya a otra felicidad: La tumba, fiel y fatal como la religión, se la entregaba muerta.

"Cruzando más los brazos sobre la borda, Morán contempló hasta perderse de vista el país que abandonab:i

"El había invocado cien veces al Destino, como a una inrencible Divinidad. Podía quedar en adelante tranquilo: La fatalidad del suyo quedaba cumplida allí”.

En vista del fracaso de Quiroga como plantador de algodón en el Chaco, y plantador de yerba mate en Misiones; en vista de la muerte de sus dos esposas, la una suicidándose y la otra muriéndose, creo, de la meningitis; y en vista de su propic viaje sin retorno cuando sale de San Ignacio algunos años más tarde bajo circunstancias análogas para irse a Buenos Aires donde se envenena, estas líneas tienen un doble significado.

Antes de esta partida final, en su casa de San Ignacio, Quiroga ordena sin prisa su último libro, Más allá, 1935, en que vuelve a las narraciones fantásticas y atormentadas de su primera época. ¿Esto tal vez revela algún presentimiento de la muerte? Por algo dió al libro tal título y puso por primer cuento el doble suicidio de Más ALLÁ. Algunas de las nárraciones en esta colección parecen haber sido escritas varios años antes, pero Quiroga sin duda las había retocado todas. Más allá describe la existencia "post-mortem" de dos amantes que se suicidan con ciantro. Quiroga también empleó el cianuro para eliminarse de la vida. La novia en MÁs allá expresa los siguientes sentimientos que bien pudieran haber sido los del autor: "No puedo decir que me sentía orgullosa de lo que iba a hacer, ni tampoco feliz de morir. Era algo más fatal, menos frenético, más sin remisión, como si desde el fon- 
do del pasado de mis abuelos, mis bisabuelos, mi infancia misma, mi primera comunión, mis ensueños, como si todo esto no hubiera tenido otra finalidad que empujarme al suicidio". Como dice Alberto Lasplaces: "Estas frases parecen ser la confesión involuntaria e indirecta de un lento drama interno que venía desgarrándolo desde muy atrás”. ( I ) Como otros muchos suicidas, Quiroga está procurando exculparse o al menos justificar espiritualmente el desesperado acto final de su existencia echando la culpa a esa misma existencia y hasta a su pasado prenatal. En otra parte encontramos esta interpretación e identificación de la vida, la muerte y el amor que ya había expresado en Pasado amor: “¡Muertos! ¿Qué absurdo! Lo que había en nosotros, más fuerte que la vida misma, continuaba viviendo con todas las esperanzas de un eterno amor". Lasplaces comenta: "La obsesión de la muerte, pero no la muerte que es un fin, y por lo tanto una clausura y un descanso, sino una prolongación etérea de la vida terrena que prolonga hasta ella sus preocupaciones y problemas, inspira estas tramas sombrías..." EL vaMPIRo vuelve sobre el mismo tema afirmando que "el amor no hace falta en la vida; pero es indispensable para golpear ante las puertas de la muerte". (1) En Las moscas, que es una réplica de Ex hombre Muerro de Los desterrados, el protagonista, después de quebrarse la columna vertebral tropezando contra un raigón, oye un zumbido de moscas que interpreta de varios modos extravagantes, finalmente identificándolo con el zumbido de su propia alma libertada: "Puedo ir aquí, allá, a este árbol, a aquella liana. Puedo ver, lejanísimo ya, como un recuerdo de remoto existir, ojos, sin parpadeo, un espantapájaros de mirar vidrioso y piernas rígidas. Del seno de esta expansión, que el sol dilata desmenuzando mi conciencia en un billón de partículas, predo alzarme y volar, volar... $\mathrm{Y}$ vuelo, y me poso con mis compa-

(I),-Loc. cit.

(2).-Varios de estos pasajes de Quiroga me recuerdan las palabras finales de la novela norteamericana, The bridge of San Luis Rey por Thornton Wilder: "There is a land of the living, and a land of the dead, and the bridge is love, the only survival, the only meaning". 
ñeras sobre el tronco caído, a los rayos del sol que prestan su fuego a nuestra obra de renovación vital". Comparado con la fría lógica de EL hombre muerto este relato es el desvarío de una mentalidad desequilibrada. El autor presenta un extremo mucho más violento en EL CONDUCTOR DEL RÁPIDO, estudio de la locura frenética, donde "se deja arrastrar por atroces delirios en que la demencia hace absurdos gestos incomprensibles". Basta este pasaje para dar una idea del conjunto: "AAmigo! ¿Usted nunca vió un hombre que se vuelve loco? Aquí está: Prrrrr! ...jOjo a la trocha 4004! Gato. - iFogonero! iVamos a palear de firme y nos comeremos la trocha 29000000003 !.. ¡Dios de la razón y de mi pobre hija! ¡Concédeme tan sólo tiempo para poner la mano sobre la palanca-blanca-piribanca, Miau!'. Al llegar al fin de este cuento el lector tiene el vago sentido de haber sido ultrajado, de haber estado en contacto con algo asqueroso. EL LLAMAdo presenta otro caso de suicidio, esta vez de una niña que se masa con una pistola al ser llamada por su padre muerto, y en EL PURITANo un hombre casado se suicida para reunirse con una estrella de la pantalla a quien había amado en la vida con una pasión puritana. SU AUSENCIA, intrigante presentación de la amnesia, es un relato relativamente normal y no pertenece a la categoría de los susodichos. En resumen, pues, podemos decir de Más allá, última obra de nuestro autor, que "amor, locura y muerte, sus temas favoritos, vuelven a enseñorearse de las páginas de este libro que tiene aspectos de predicción y de testamento, al mismo tiempo que de reconquista de la sustancia esencial". (I)' Y comprendemos por qué el pobre solitario "adquirió una dosis de cianuro y hacia la madrugada, en la soledad de su pieza, sin luz y sin testigos, se envenenó". Fué el acto lógico y desesperado de un espíritu lúgubre, sensible y huraño que las circunstancias de la vida y la propia sensibilidad e inmenso incumplido amor llevaron inexorablemente a la locura

(1).-Alberto Lasplaces, loc. cit. 
o loco desengaño, dándole la sensación imperativa de tener que librarse de las cadenas de esta existencia para buscar en el más allá un amor eterno. (2)

John A. Crow.

(2).-Nota del autor de este estudio: La mayoría de las obras de Quiroga demuestran cierta tendencia hacia lo anormal, pero yo he procurado abreviar mi artículo todo lo posible tomando solamente los ejemplos más patentẹs. 
